\title{
In vitro T Cell Reactivity in Nickel Allergy: Comparison of T Cell Clonality, Cytokine Expression and Mediator Production
}

\author{
Peter Thomas Burkhard Summer Christian Sander Bernhard Przybilla \\ Klinik für Dermatologie und Allergologie, Ludwig-Maximilians-Universität, München, Deutschland
}

\section{Key Words}

Lymphocytes · Nickel allergy $\cdot$ T cell receptor - IL-4 . IFN- $\gamma \cdot$ PCR

\section{Introduction}

Apart from typical symptoms of nickel contact allergy, there are also unusual manifestations, e.g. pseudolymphoma formation, airborne contact dermatitis or implantassociated intolerance reactions [1]. Delayed-type hypersensitivity to nickel is reflected by eczematous reactions upon patch test and by an antigen-specific $\mathrm{T}$ lymphocyte proliferation in vitro [2-4]. We now assessed to what extent peripheral blood mononuclear cells (PBMC) from nickel-allergic and from nonallergic individuals would proliferate in response to nickel stimulation in vitro. In addition, the production of IL-4 and IFN- $\gamma$ in these cultures was monitored by RT-PCR of the respective mRNA and by immunoassay of the supernatants. In addition, we examined with a PCR analysis of the several families of the $\mathrm{T}$ cell receptor gamma (TCR- $\gamma$ ) gene, if instead of being randomly stimulated $\mathrm{T}$ cells would show a clonal expansion pattern.

\section{Materials and Methods}

Suspensions of PBMC were obtained by centrifugation from the peripheral blood of 10 nickel-allergic individuals and 5 controls not allergic to nickel. The subsequent cell culture was performed either with complete $10 \% \mathrm{AB}$ serum containing medium alone or with the addition of phytohemagglutinin (PHA; $2.4 \mu \mathrm{g} / \mathrm{ml}$ ), tetanus toxoid (TT; $5.0 \mu \mathrm{g} / \mathrm{ml})$ or nickel sulfate $\left(10^{-4} M, 10^{-5} M\right)$. On day 5 a proliferative response was assessed by ${ }^{3} \mathrm{H}$-thymidine uptake and expressed as stimulation index.

Released IL-4 [detection limit (d.1.): $1.5 \mathrm{pg} / \mathrm{ml}$ ] and IFN- $\gamma$ (d.1.: $5.0 \mathrm{pg} / \mathrm{ml}$ ) in the supernatants were measured by ELISA. Samples below d.1. were set to $0.75 \mathrm{pg} / \mathrm{ml}$ (IL-4) and $2.5 \mathrm{pg} / \mathrm{ml}$ (IFN- $\gamma$ ). To evaluate the respective actual mRNA levels extraction by the phenol/ chloroform method was done. RT-PCR (IL- 4 , IFN- $\gamma$, $\beta$-actin) was applied according to standard protocols. To cover the range of the TCR- $\gamma$ chain we used a combination of primers for the main groups of the variable region $(\mathrm{V} \gamma 1-8, \mathrm{~V} \gamma 9, \mathrm{~V} \gamma 10, \mathrm{~V} \gamma 11)$ and a primer mix, which contains several sequences of different joining regions. The TCR region $\mathrm{V} \gamma 1-8$ was detected by the consensus primer $\mathrm{V} \gamma 2$. PCR products were separated on a $6 \%$ polyacrylamide gel and photographed after staining with ethidium bromide. Clonal expansion of few specifically reacting $T$ cell populations would result in defined bands in contrast to a 'smear' in the case of polyclonal TCR- $\gamma$ rearangement.

\begin{tabular}{ll}
\hline KARGER & ( ) 2001 S. Karger AG, Basel \\
Fax +4161306 1234 & 1018-2438/01/1243-0292\$17.50/0 \\
$\begin{array}{l}\text { E-Mail karger@karger.ch } \\
\text { www.karger.com }\end{array}$ & $\begin{array}{l}\text { Accessible online at: } \\
\text { www.karger.com/journals/iaa }\end{array}$
\end{tabular}

Correspondence to: PD Dr. P. Thomas

Klinik und Poliklinik für Dermatologie und Allergologie

Ludwig-Maximilians-Universität München, Frauenlobstrasse 9-11

D-80337 München (Germany), Tel. +49 8951606314

Fax+498951606312, E-Mail Peter.Thomas@derma.med.uni-muenchen.de 


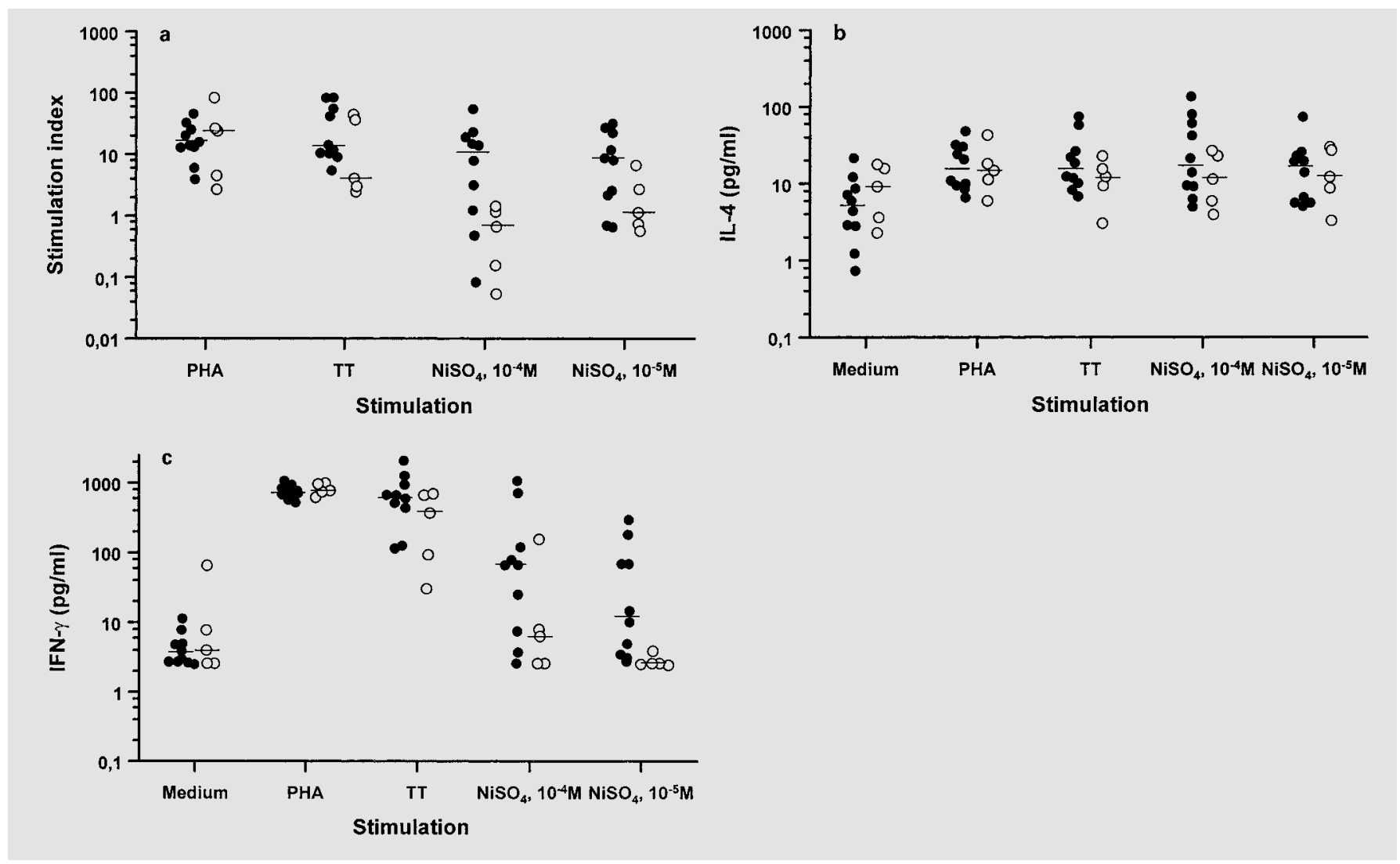

Fig. 1. a Proliferative response (stimulation index) from PBMC of 10 nickel-allergic and 5 nonallergic individuals. b Production of IL-4 from PBMC of 10 nickel-allergic and 5 nonallergic individuals. d.1. was $1.5 \mathrm{pg} / \mathrm{ml}$ (samples below d.l. are expressed as $0.75 \mathrm{pg} / \mathrm{ml}$. c Production of IFN- $\gamma$ from PBMC of 10 nickel-allergic and 5 nonallergic individuals. d.l. was $5.0 \mathrm{pg} / \mathrm{ml}$ (samples below d.l. are expressed as $2.5 \mathrm{pg} / \mathrm{ml}$ ). $\bullet=$ Nickel-allergic; $\bigcirc=$ nonallergic.

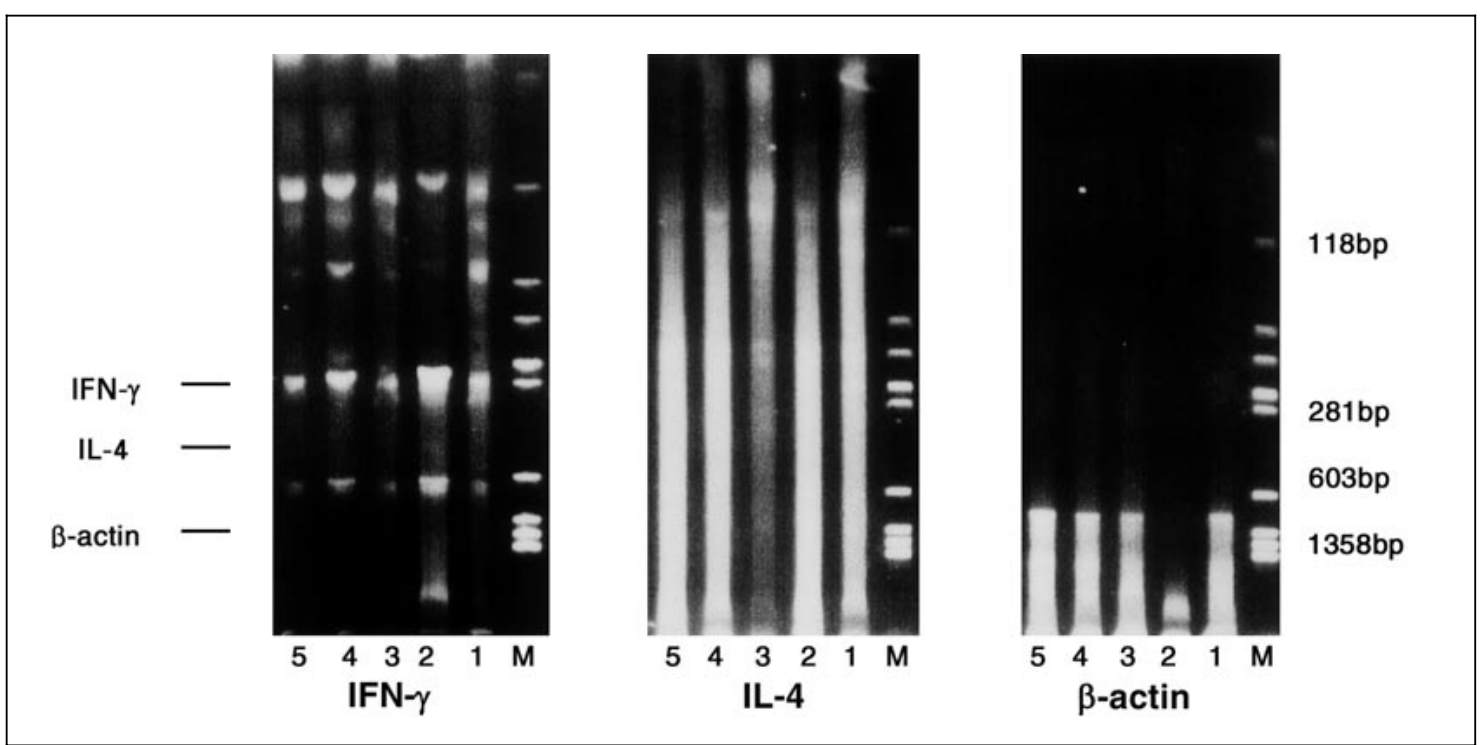

Fig. 2. RT-PCR products of mRNA from cell culture series (here nickel-allergic individual No. 10). 1 = Medium; $2=$ PHA; $3=\mathrm{TT} ; 4=\mathrm{NiSO}_{4} 10^{-4} M ; 5=\mathrm{NiSO}_{4} 10^{-5} M ; \mathrm{M}=$ DNA marker. 
Table 1. Analysis of TCR- $\gamma$ specificity upon medium or allergen stimulation (clonality)

\begin{tabular}{llllll}
\hline $\begin{array}{l}\text { Allergic } \\
\text { individuals }\end{array}$ & $\begin{array}{l}\text { Medium } \\
\text { alone }\end{array}$ & PHA & $\mathrm{TT}$ & $\begin{array}{l}\mathrm{NiSO}_{4} \\
10^{-4} M\end{array}$ & $\begin{array}{l}\mathrm{NiSO}_{4} \\
10^{-5} M\end{array}$ \\
\hline 1 & - & - & $\mathrm{V} \gamma 11$ & - & - \\
2 & - & - & $\mathrm{V} \gamma 11$ & $\mathrm{~V} \gamma 10$ & - \\
3 & - & - & $\mathrm{V} \gamma 11$ & $\mathrm{~V} \gamma 10$ & $\mathrm{~V} \gamma 10$ \\
4 & - & - & $\mathrm{V} \gamma 9$ & $\mathrm{~V} \gamma 9$ & $\mathrm{~V} \gamma 9$ \\
5 & - & - & $\mathrm{V} \gamma 11$ & $\mathrm{~V} \gamma 2, \mathrm{~V} \gamma 11$ & - \\
6 & $\mathrm{~V} \gamma 9$ & $\mathrm{~V} \gamma 9$ & $\mathrm{~V} \gamma 9$ & $\mathrm{~V} \gamma 9$ & $\mathrm{~V} \gamma 9$ \\
7 & - & - & - & $\mathrm{V} \gamma 10$ & - \\
8 & - & - & $\mathrm{V} \gamma 11$ & $\mathrm{~V} \gamma 10$ & $\mathrm{~V} \gamma 10$ \\
9 & - & - & - & - & - \\
10 & - & - & $\mathrm{V} \gamma 9$ & $\mathrm{~V} \gamma 2$ & $\mathrm{~V} \gamma 2$ \\
\hline Controls & & & & & \\
11 & - & - & $\mathrm{V} \gamma 11$ & - & $\mathrm{V} \gamma 11$ \\
12 & - & - & $\mathrm{V} \gamma 9$ & $\mathrm{~V} \gamma 2, \mathrm{~V} \gamma 9$ & $\mathrm{~V} \gamma 9$ \\
13 & - & - & $\mathrm{V} \gamma 9$ & - & - \\
14 & $\mathrm{~V} \gamma 10$ & $\mathrm{~V} \gamma 2$ & $\mathrm{~V} \gamma 10$ & $\mathrm{~V} \gamma 2$ & - \\
15 & - & - & - & $\mathrm{V} \gamma 11$ & $\mathrm{~V} \gamma 10$ \\
\hline
\end{tabular}

- = No preferential TCR- $\gamma$ expression; $\mathrm{V} \gamma 2, \mathrm{~V} \gamma 9, \mathrm{~V} \gamma 10, \mathrm{~V} \gamma 11=$ predominant TCR- $\gamma$ family.

\section{Results and Discussion}

PBMC of nickel-allergic patients proliferated upon the addition of nickel sulfate to a varying degree. Cells of the different blood donors - some of whom had actual eczema - showed a baseline IL-4 production. In contrast, IFN- $\gamma$ in the supernatants was enhanced in nickel-stimulated cultures of allergic individuals (fig. 1). This was reflected by the predominant detection of IFN- $\gamma$-specific mRNA on day 5 (tables 1, 2, fig. 2). Despite most T cells showing surface expression of TCR- $\alpha \beta$, the analysis of the concomitant rearrangement of TCR- $\gamma$ gene makes it possible to evaluate the array of T lymphocytes with regard to clonality. Here the analysis of the TCR- $\gamma$ spectrum in the specifically stimulated cultures showed clonal expansion (tables 1,2) in contrast to the unspecific, broad stimulation by PHA. This would point to a rather specific response in sensitized individuals in contrast to random $T$ cell activation. Thus, the combined analysis of proliferative response in vitro, mRNA characterization and potential clonal TCR- $\gamma$ rearrangement $[5,6]$ could further help to identify specific antigen/allergen-induced $\mathrm{T}$ cell activation.

Table 2. Qualitative evaluation (band intensity) of RT-PCR products (IL-4/IFN- $\gamma$ )

\begin{tabular}{|c|c|c|c|c|c|c|c|c|c|c|}
\hline \multirow{2}{*}{$\begin{array}{l}\text { Allergic } \\
\text { individuals }\end{array}$} & \multicolumn{2}{|c|}{ Medium alone } & \multicolumn{2}{|l|}{ PHA } & \multicolumn{2}{|l|}{$\mathrm{TT}$} & \multicolumn{2}{|c|}{$\mathrm{NiSO}_{4} 10^{-4} M$} & \multicolumn{2}{|c|}{$\mathrm{NiSO}_{4} 10^{-5} M$} \\
\hline & IFN- $\gamma$ & IL-4 & IFN- $\gamma$ & IL-4 & IFN- $\gamma$ & IL-4 & IFN- $\gamma$ & IL-4 & IFN- $\gamma$ & IL-4 \\
\hline 1 & - & - & +++ & - & ++ & - & ++ & - & +++ & - \\
\hline 2 & - & - & +++ & ++ & ++ & - & +++ & - & ++ & + \\
\hline 3 & - & - & +++ & + & + & - & +++ & - & +++ & - \\
\hline 4 & - & - & +++ & - & - & - & ++ & - & +++ & - \\
\hline 5 & - & - & +++ & - & +++ & - & +++ & - & +++ & - \\
\hline 6 & + & - & +++ & - & ++ & - & +++ & - & +++ & + \\
\hline 7 & - & - & +++ & - & ++ & - & +++ & - & +++ & - \\
\hline 8 & + & - & +++ & + & + & - & ++ & - & ++ & - \\
\hline 9 & + & - & +++ & - & ++ & + & +++ & - & + & - \\
\hline 10 & ++ & - & +++ & - & + & - & + & - & ++ & - \\
\hline \multicolumn{11}{|l|}{ Controls } \\
\hline 11 & - & - & +++ & + & - & - & + & - & - & - \\
\hline 12 & - & - & +++ & - & + & - & + & - & + & - \\
\hline 13 & - & - & +++ & + & ++ & - & - & - & + & - \\
\hline 14 & - & - & +++ & - & - & - & + & - & - & - \\
\hline 15 & - & - & +++ & - & - & - & - & - & + & - \\
\hline
\end{tabular}

mRNA was extracted from unstimulated (medium alone) or allergen stimulated cultures. $-=$ Absent; $+=$ visible band; ++ = strong band; +++ = very strong expression. Evaluation was done with reference to $\beta$-actin expression. 


\section{References}

1 Möller H: Nickel dermatitis: Problems solved and unsolved. Contact Dermatitis 1990;23: 217-220

2 Cederbrant K, Hultman P, Marcusson JA, Tibbling L: In vitro lymphocyte proliferation as compared to patch test using gold, palladium and nickel. Int Arch Allergy Immunol 1997; 112:212-217.

3 Kapsenberg ML, Wierenga EA, Stiekema F, Tiggelman A, Bos JD: Th1 lymphokine production profile from nickel-specific CD4+ Tlymphocyte clones from nickel contact allergic and non-allergic individuals. J Invest Dermatol 1992;98:59-63.
4 Mitchison NA: Specialization, tolerance, memory, competition, latency, and strife among T cells. Annu Rev Immunol 1992;10:1-12.

5 Thomas P, Thomas M, Summer B, Naumann T, Sander CA, Przybilla B: Intolerance of osteosynthesis material: Evidence of dichromate contact allergy with concomitant oligoclonal Tcell infiltrate and TH1-type cytokine expression in the peri-implantar tissue. Allergy 2000; 55:969-972.

6 Werfel T, Hentschel M, Kapp A, Renz H: Dichotomy of blood- and skin-derived IL-4producing allergen-specific $\mathrm{T}$ cells and restricted $\mathrm{V} \beta$ repertoire in nickel-mediated contact dermatitis. J Immunol 1997;158:25002505 . 